\title{
Prevalence of intestinal protozoa infection among school-aged children on Pemba Island, Tanzania, and effect of single-dose albendazole, nitazoxanide and albendazole-nitazoxanide
}

\author{
Benjamin Speich ${ }^{1,2}$, Hanspeter Marti ${ }^{2,3}$, Shaali M Ame ${ }^{4}$, Said M Ali4, Isaac I Bogoch5 ${ }^{5}$ Jürg Utzinger ${ }^{2,6}$,
} Marco Albonico ${ }^{7}$ and Jennifer Keiser ${ }^{1,2^{*}}$

\begin{abstract}
Background: Pathogenic intestinal protozoa infections are common in school-aged children in the developing world and they are frequently associated with malabsorption syndromes and gastrointestinal morbidity. Since diagnosis of these parasites is difficult, prevalence data on intestinal protozoa is scarce.

Methods: We collected two stool samples from school-aged children on Pemba Island, Tanzania, as part of a randomized controlled trial before and 3 weeks after treatment with (i) single-dose albendazole (400 mg); (ii) single-dose nitazoxanide (1,000 mg); (iii) nitazoxanide-albendazole combination (1,000 mg-400 mg), with each drug given separately on two consecutive days; and (iv) placebo. Formalin-fixed stool samples were examined for the presence of intestinal protozoa using an ether-concentration method to determine the prevalence and estimate cure rates (CRs).

Results: Almost half (48.7\%) of the children were diagnosed with at least one of the (potentially) pathogenic protozoa Giardia intestinalis, Entamoeba histolytica/E. dispar and Blastocystis hominis. Observed CRs were high for all treatment arms, including placebo. Nitazoxanide showed a significant effect compared to placebo against the non-pathogenic protozoon Entamoeba coli.

Conclusions: Intestinal protozoa infections might be of substantial health relevance even in settings where they are not considered as a health problem. Examination of a single stool sample with the ether-concentration method lacks sensitivity for the diagnosis of intestinal protozoa, and hence, care is indicated when interpreting prevalence estimates and treatment effects.
\end{abstract}

Keywords: Intestinal protozoa, Blastocystis hominis, Entamoeba histolytica/E. dispar, Giardia intestinalis, Albendazole, Nitazoxanide, Ether-concentration method

\section{Background}

Infection with pathogenic intestinal protozoa (e.g. Entamoeba histolytica and Giardia intestinalis) result in considerable gastrointestinal morbidity, malnutrition and mortality worldwide, particularly among young children in developing countries [1,2]. It has been estimated that

\footnotetext{
*Correspondence: jennifer.keiser@unibas.ch

'Department of Medical Parasitology and Infection Biology, Swiss Tropical and Public Health Institute, P.O. Box, CH-4002 Basel, Switzerland

2University of Basel, P.O. Box, CH-4003 Basel, Switzerland

Full list of author information is available at the end of the article
}

E. histolytica, the causative agent of amoebiasis, kills between 40,000 and 100,000 people each year; hence, is one of the deadliest parasitic infections worldwide [2,3]. In the People's Republic of China alone, G. intestinalis affects an estimated 28.5 million people every year [1]. The prevalence of G. intestinalis has been estimated at $2-3 \%$ in the industrialized world and $20-30 \%$ in developing countries [4]. Cryptosporidium spp. is another major causal agent of diarrhoea, primarily affecting immunocompromised individuals such as those infected with HIV [3,5,6]. Blastocystis hominis is a common additional anaerobic intestinal protozoon and 
its pathogenicity is still under debate [7-9]. Lack of access to clean water, sanitation and hygiene are strong drivers for infection with intestinal protozoa [10-12].

Several drugs are currently available to treat intestinal protozoa infections. Most commonly used are 5nitroimidazole compounds, including metronidazole, tinidazole, ornidazole and secnidazole [13]. Alternative effective agents, when given as multiple doses, include nitazoxanide and albendazole [14-16].

Information on the prevalence of intestinal protozoa infections is scarce and little data are available from subSaharan Africa. For example, to the best of our knowledge, the prevalence of intestinal protozoa infections on Pemba Island has been assessed only twice and these investigations date back to 1984 and $1992[17,18]$. In the 1984 study, the prevalence of G. intestinalis and E. histolytica among children and adults combined were $5.6 \%$ and $3.1 \%$, respectively. Prevalences of $35.6 \%, 4.4 \%, 2.9 \%, 0.7 \%$ and $0.7 \%$ were reported for Entamoeba coli, Endolimax nana, Chilomastix mesnili, Entamoeba hartmanni and Iodamoeba bütschlii, respectively (Figure 1) [17]. The study conducted in 1992 reported prevalences of $25.4 \%$ for E. histolytica and $6.6 \%$ for G. intestinalis among children aged 9-17 years [18].

The study reported here was integrated in a randomized controlled trial carried out in school-aged children on Pemba Island, Tanzania in mid-2011 to asses the efficacy and safety of single-dose nitazoxanide and albendazole and a nitazoxanide-albendazole combination against Trichuris trichiura and other soil-transmitted helminth infections [19]. The aim of the present work was to evaluate the prevalence of intestinal protozoa infections in these children and to determine whether the study drugs had an effect on intestinal protozoa. Throughout the study, the ether-concentration method was used on formalin-fixed stool samples.

\section{Methods}

\section{Ethics statement}

Our randomized controlled trial was approved by the ethics committee of Basel (EKBB; reference no. 225/10), and the Ministry of Health and Social Welfare of Zanzibar (ZAMREC; reference no. 0001/010) and is registered at Current Controlled Trials (trial identifier: ISRCTN08336605). Participating in the trial required written informed consent from parents or legal guardians and oral assent from children. Parents and children were counseled that participation is voluntary and withdrawal was possible at any time without specification of reasons and further obligations. At the end of the trial, all children who were diagnosed with soil-transmitted helminths received albendazole (single oral dose of $400 \mathrm{mg}$ ).

\section{Study area and design}

Details of the study area, population and procedures have been described previously [19]. In brief, the study was carried out in June and July 2011 in children aged 7-15 years attending two schools (Wawi and Al-Sadik) located within a radius of $10 \mathrm{~km}$ from Chake Chake, the main city of Pemba Island. The study was a randomized controlled trial with four treatment arms: (i) single-dose albendazole (400 mg); (ii) single-dose nitazoxanide (1,000 mg); (iii) nitazoxanide-albendazole combination (1,000 mg-400 mg) with each drug given separately on two consecutive days; and (iv) placebo. Of the 657 children, who completed the trial, 550 (83.7\%) had formalin-fixed stool samples before and after treatment and were therefore included in the present study (treatment arms: (i) $\mathrm{n}=136$, (ii) $\mathrm{n}=145$, (iii) $\mathrm{n}=117$, and (iv) $\mathrm{n}=152$; Wawi school: $\mathrm{n}=404$, Al-Sadik school: $\mathrm{n}=146)$.

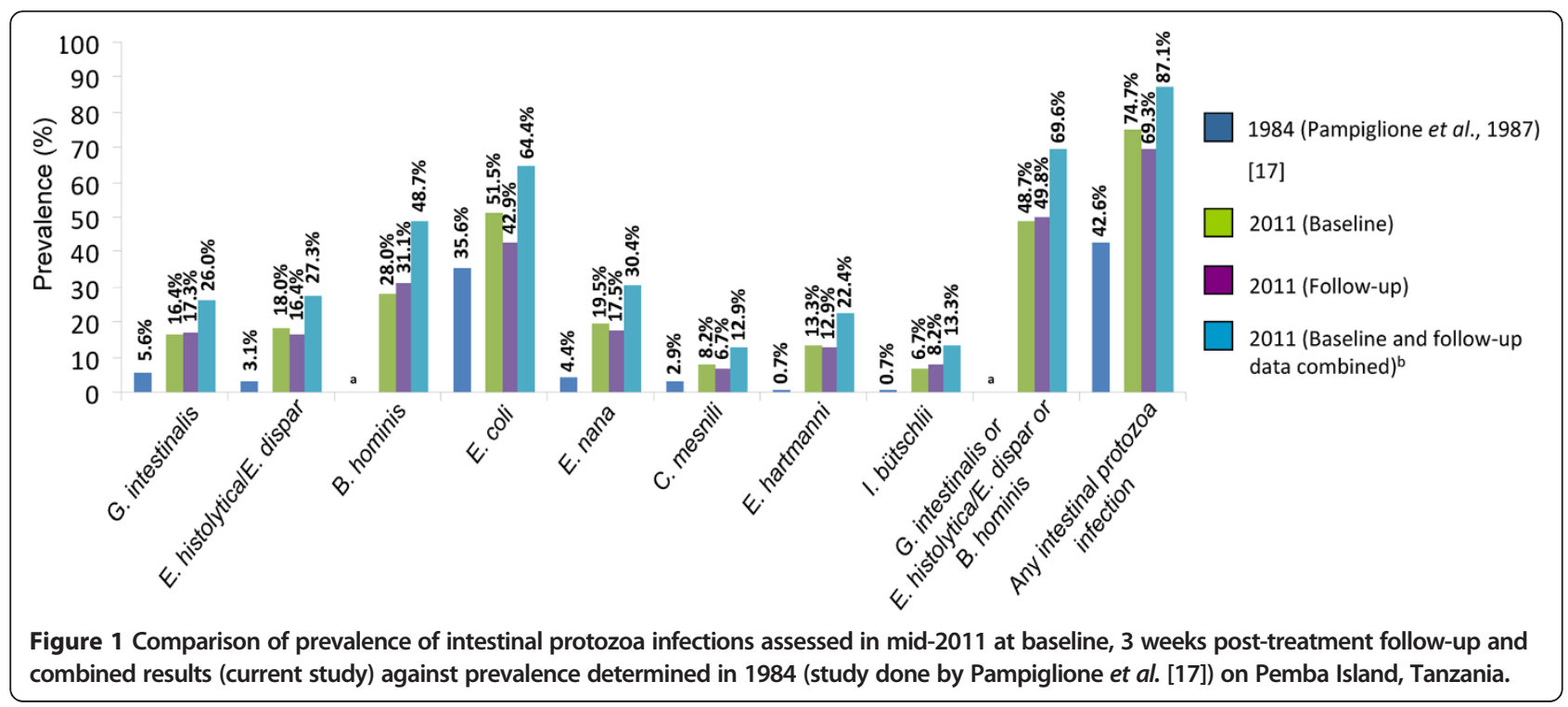




\section{Procedures for the diagnosis of intestinal protozoa}

Approximately $2 \mathrm{~g}$ of stool were fixed in $10 \mathrm{ml}$ of $5 \%$ formalin in Falcon tubes labeled with unique identifiers. Stool samples were thoroughly broken down and homogenized with a wooden spatula. The formalin-fixed samples were transferred to the Swiss Tropical and Public Health Institute (Basel, Switzerland) and examined within 10 months.

The fixed stool samples were processed with an etherconcentration method [20,21]. Briefly, the homogenized stool sample was filtered through a medical gauze into a new tube and then centrifuged for $1 \mathrm{~min}$ at $500 \mathrm{~g}$. The supernatant was discarded. To the remaining pellet, $7 \mathrm{ml}$ of physiological $\mathrm{NaCl}$-solution and $2-3 \mathrm{ml}$ of diethyl ether was added. Tubes were shaken and centrifuged again for 3 min at $500 \mathrm{~g}$. The upper three layers were discarded. The entire sediment was examined by experienced laboratory technicians under a microscope for soil-transmitted helminths at a magnification of $100 \times$, and intestinal protozoa at a magnification of $400 \times$ or $500 \times$ using oil immersion. The current analysis focuses on intestinal protozoa, including the pathogenic intestinal protozoa G. intestinalis and E. histolytica/E. dispar (of note, these two Entamoeba species cannot be differentiated by microscopy [22]); the potentially pathogenic protozoon $B$. hominis, and the nonpathogenic protozoa E. coli, E. hartmanni, E. nana, C. mesnili and I. bütschlii [7-9,23]. Cryptosporidium spp. was not included, since it cannot easily be detected with the formalin-ether concentration technique, and would have required staining with the modified Ziehl-Neelsen method [24]. Infection intensities were classified as follows: (i) negative (no cysts or trophozoites in the entire sediment); (ii) light (one to five cysts or trophozoites per slide); (iii) moderate (one cyst or trophozoite per observation field at the $400 \times$ or $500 \times$ magnification); and (iv) heavy (more than one cyst or trophozoite per observation field) $[21,25]$.

\section{Statistical analysis}

Data were double-entered into an Excel file (Microsoft 2010) and cross-checked. Statistical analysis was performed using Stata version 10.1 (StataCorp; College Station, USA).

The prevalence of intestinal protozoa was determined before treatment (baseline) and at the 3-week post-treatment follow-up. Differences between prevalence at baseline and follow-up were assessed using $x^{2}$ test. In the baseline prevalence analyses, the odds of being infected with a specific intestinal protozoon species for boys compared to girls and for children from Wawi compared to Al-Sadik school was calculated using logistic regression.

Cure rates (CRs) were estimated for each intestinal protozoon species for the different treatment arms as the percentage of positive children at baseline diagnosed negative after treatment. Differences in CRs among treatment arms were examined using logistic regressions.
Reduction of infection intensity was assessed as the difference in infection intensities before and after treatment among all individuals (negative individuals before treatment were included in the analysis). Mean of differences in infection intensities was calculated for each treatment arm and for each intestinal protozoon species together with 95\% confidence intervals (CIs). Decrease in infection intensity was assumed as significant when $95 \%$ CI was below 0 .

\section{Results}

\section{Prevalence and intensity of intestinal protozoa infection}

Based on the analysis of one formalin-fixed stool sample per child at baseline, $74.7 \%$ of the children harboured at least one intestinal protozoa species. About half of the children $(48.7 \%)$ were infected with at least one of the three (potentially) pathogenic intestinal protozoa. The prevalence of $E$. histolytica/E. dispar and G. intestinalis was $18.0 \%$ and $16.4 \%$, respectively before treatment (Table 1 ). The potentially pathogenic intestinal protozoon B. hominis was diagnosed in $28.0 \%$ of the children. Prevalences for the other intestinal protozoa were $51.5 \%$ for E. coli, $19.5 \%$ for E. nana, $13.3 \%$ for E. hartmanni, $8.2 \%$ for C. mesnili and $6.7 \%$ for I. bütschlii. For the pathogenic intestinal protozoon G. intestinalis, $44.4 \%$, 38.9\% and $16.7 \%$ of the infections were classified as light, moderate and heavy, respectively. The infection intensities of other intestinal protozoa species are listed in Table 1.

Examination of the follow-up stool samples revealed the following prevalences: $49.8 \%$ of children remained infected with at least one of the three (potentially) pathogenic intestinal protozoa and $69.3 \%$ of the children were found positive for at least one intestinal protozoa. The prevalence of G. intestinalis, E. histolytica/E. dispar and B. hominis at the 3-week post-treatment follow-up was $17.3 \%, 16.4 \%$ and $31.1 \%$, respectively (Figure 1). Prevalence for the nonpathogenic intestinal protozoa species were $42.9 \%$ for E. coli, $17.5 \%$ for E. nana, $12.9 \%$ for $E$. hartmanni, $8.2 \%$ for I. bütschlii and $6.7 \%$ for $C$. mesnili. According to the $x^{2}$ test, only E. coli $(p=0.004)$ and all intestinal protozoa combined $(p=0.046)$ showed significantly lower prevalence at follow-up compared to baseline.

The odds of being infected with any intestinal protozoa at baseline was significantly higher for girls than boys (odds ratio $(\mathrm{OR})=1.97 ; 95 \% \mathrm{CI} 1.32-2.93$ ) (Table 2). There was a trend $(p<0.1)$ that girls were at a higher odds of being infected with $E$. coli than boys $(\mathrm{OR}=1.40$; $95 \%$ CI $1.00-$ 1.96). Children from Wawi school had significantly lower odds of being infected with $B$. hominis ( $\mathrm{OR}=0.64 ; 95 \% \mathrm{CI}$ $0.43-0.97), \quad$ E. nana (OR $=0.63 ; 95 \%$ CI $0.40-1.00)$ and I. bütschlii ( $\mathrm{OR}=0.50$; 95\% CI $0.25-1.00)$ considering only the baseline stool sample than children from Al-Sadik. Additionally, there was a trend $(p<0.1)$ that children from Wawi school had a lower odds of being infected with G. intestinalis $(\mathrm{OR}=0.66 ; 95 \% \mathrm{CI} 0.41-1.07)$ and any of 
Table 1 Baseline characteristics of included school-aged children on Pemba Island in mid-2011 with regard to intestinal protozoa infection

\begin{tabular}{|c|c|c|c|}
\hline \multirow[t]{2}{*}{ Characteristic, intestinal protozoa } & $\mathrm{N}$ (prevalence in \%) & \multirow[t]{2}{*}{ Girls (\%)/boys (\%) } & \multirow{2}{*}{$\begin{array}{c}\text { Wawi (\%)/Al-Sadik } \\
\text { school (\%) }\end{array}$} \\
\hline & low (\%)/moderate (\%)/heavy (\%) & & \\
\hline No. of children tested & 550 & $271 / 279$ & $404 / 146$ \\
\hline \multirow[t]{2}{*}{ G. intestinalis } & $90(16.4)$ & $37(13.7) / 53(19.0)$ & $59(14.6) / 31(21.1)$ \\
\hline & $40(44.4) / 35(38.9) / 15(16.7)$ & & \\
\hline \multirow[t]{2}{*}{ E. histolytica/E. dispar } & $99(18.0)$ & $53(19.6) / 46(16.5)$ & $77(19.1) / 22(15.1)$ \\
\hline & $57(57.6) / 33(33.3) / 9(9.1)$ & & \\
\hline \multirow[t]{2}{*}{ B. hominis } & $154(28.0)$ & $73(26.9) / 81(29.0)$ & $103(34.9) / 51(25.5)$ \\
\hline & $125(81.2) / 25(16.2) / 4(2.6)$ & & \\
\hline \multirow[t]{2}{*}{ E. coli } & $283(51.5)$ & $151(55.7) / 132(47.3)$ & $210(52.0) / 73(50.0)$ \\
\hline & $101(35.7) / 104(36.7) / 78(27.6)$ & & \\
\hline \multirow[t]{2}{*}{ E. nana } & $107(19.5)$ & $58(21.4) / 49(17.6)$ & $71(17.6) / 36(24.7)$ \\
\hline & $62(57.9) / 40(37.4) / 5(4.7)$ & & \\
\hline \multirow[t]{2}{*}{ E. hartmanni } & $73(13.3)$ & $38(14.0) / 35(12.0)$ & $52(12.9) / 21(14.4)$ \\
\hline & $57(78.1) / 14(19.2) / 2(2.7)$ & & \\
\hline \multirow[t]{2}{*}{ C. mesnili } & $45(8.2)$ & $27(6.5) / 18(10.0)$ & $31(7.7) / 14(9.6)$ \\
\hline & $21(46.7) / 18(40.0) / 6(13.3)$ & & \\
\hline \multirow[t]{2}{*}{ I. bütschlii } & $37(6.7)$ & $18(6.6) / 19(6.8)$ & $22(5.4) / 15(10.3)$ \\
\hline & $31(83.8) / 5(13.5) / 1(2.7)$ & & \\
\hline G. intestinalis or E. histolytica/E. dispar or B. hominis & $268(48.7)$ & $135(49.8) / 133(47.8)$ & $187(46.3) / 81(55.5)$ \\
\hline Any intestinal protozoa & $411(74.4)$ & $219(80.8) / 192(68.8)$ & $297(73.5) / 114(78.1)$ \\
\hline
\end{tabular}

the three (potentially) pathogenic intestinal protozoa combined $(\mathrm{OR}=0.68 ; 95 \%$ CI $0.47-1.00)$.

\section{Effect of antiparasitic treatment against intestinal protozoa}

Observed CRs were moderate to high for all intestinal protozoa regardless of the treatments administered

Table 2 Odds ratios (OR) of being infected with intestinal protozoa among school-aged children on Pemba Island in mid-2011, as assessed by logistic regression

\begin{tabular}{|c|c|c|}
\hline Intestinal protozoa & $\begin{array}{l}\text { OR girls vs. } \\
\text { boys }(95 \% \mathrm{Cl})\end{array}$ & $\begin{array}{l}\text { OR Wawi vs. Al-Sadik } \\
\text { school }(95 \% \mathrm{CI})\end{array}$ \\
\hline G. intestinalis & $0.67(0.44-1.11)$ & $0.66(0.41-1.07)$ \\
\hline E. histolytica/E. dispar & $1.21(0.78-1.87)$ & $1.30(0.77-2.19)$ \\
\hline B. hominis & $0.94(0.64-1.37)$ & $0.64(0.43-0.97)^{*}$ \\
\hline E. coli & $1.40(1.00-1.96)$ & $1.04(0.71-1.53)$ \\
\hline E. nana & $1.34(0.87-2.05)$ & $0.63(0.40-1.00)^{*}$ \\
\hline E. hartmanni & $1.15(0.70-1.89)$ & $0.87(0.50-1.50)$ \\
\hline C. mesnili & $1.65(0.88-3.09)$ & $0.74(0.38-1.44)$ \\
\hline I. bütschlii & $1.04(0.53-2.04)$ & $0.50(0.25-1.00)^{*}$ \\
\hline $\begin{array}{l}\text { G. intestinalis or E. histolytical } \\
\text { E. dispar or B. hominis }\end{array}$ & $1.13(0.81-1.58)$ & $0.68(0.47-1.00)$ \\
\hline Any intestinal protozoa & $1.97(1.32-2.93)^{*}$ & $0.72(0.46-1.14)$ \\
\hline
\end{tabular}

$\mathrm{Cl}$, confidence interval.
(Table 3). The highest CR was observed for the albendazole-nitazoxanide combination against $E$. nana (CR 91.3\%; 95\% CI 78.8-100.0\%). The lowest CR among the antiparasitics tested was documented for single-dose albendazole against E. coli (CR 33.3\%; 95\% CI 22.4-44.3\%). Note that the group of children receiving placebo had moderate to high CRs against intestinal protozoa infections. Comparing the outcomes among treatment arms using logistic regression revealed that single-dose nitazoxanide had a significant effect on $E$. coli $(\mathrm{OR}=0.35 ; 95 \%$ CI 0.18-0.68). Furthermore, we observed a trend $(p<0.1)$ with the combination of nitazoxanide plus albendazole against E. nana (OR $=0.18 ; 95 \%$ CI 0.02-1.27). All other results of the logistic regressions revealed p-values above 0.1 ; hence, there was no significant effect compared to placebo.

Comparing the mean intensity of intestinal protozoa infection before and 3 weeks after treatment in the different arms revealed no significant effect for most of the assessed intestinal protozoa (results not shown). The only significant reductions of infection intensity (95\% CI below 0) were observed in the albendazole-nitazoxanide combination against $E$. histolytica/E. dispar $(-0.21 ; 95 \%$ CI -0.34 to -0.09$)$ and E. coli $(-0.37 ; 95 \%$ CI -0.58 to -0.16$)$ and in the nitazoxanide single-dose treatment against $E$. coli infection intensity $(-0.37$; $95 \%$ CI -0.58 to -0.16$)$. 
Table 3 Effect of albendazole, nitazoxanide, sequentially administered albendazole-nitazoxanide combination, and placebo against intestinal protozoa infections among school-aged children on Pemba Island in mid-2011

\begin{tabular}{lcccc}
\hline Characteristic & $\begin{array}{c}\text { Single-dose } \\
\text { albendazole }\end{array}$ & $\begin{array}{c}\text { Single-dose } \\
\text { nitazoxanide }\end{array}$ & $\begin{array}{c}\text { Nitazoxanide- } \\
\text { albendazole combination }\end{array}$ \\
\hline G. intestinalis & & & & Placebo \\
No. of infected children & 25 & 21 & 19 & 11 \\
No. of children not cured after treatment & 10 & 57.1 & 42.1 & 12 \\
CR, \% (95\% Cl) & 60.0 & $(34.1-80.2)$ & $(17.7-66.6)$
\end{tabular}

\section{E. histolytica/E. dispar}

No. of infected children

$\begin{array}{cc}23 & 23 \\ 10 & 9 \\ 56.5 & 60.9\end{array}$

No. of children not cured after treatment

10

CR, \% (95\% Cl)

\section{B. hominis}

No. of infected children

No. of children not cured after treatment

$\begin{array}{cc}35 & 37 \\ 15 & 16 \\ 57.1 & 56.8\end{array}$

(50.3-85.2)

(31.9-77.1)

CR, \% (95\% Cl)

33
10
69.7
67.3

53.1-86.2)

E. coli

No. of infected children

No. of children not cured after treatment

CR, \% (95\% Cl)

$\begin{array}{cc}75 & 82 \\ 50 & 38 \\ 33.3 & 53.7 \\ (22.4-44.3) & (42.6-64.7)\end{array}$

56
27
51.8

$(53.7-81.0)$

\section{E. hartmanni}

No. of infected children

No. of children not cured after treatment

CR, \% (95\% Cl)

$\begin{array}{cc}14 & 22 \\ 5 & 5 \\ 64.3 & 773\end{array}$

(38.3-65.3)
70
50
28.6

$(17.7-39.4)$

(35.6-93.0)

(58.3-96.3)

18
4
778

19

7

(56.5-99.1)

(39.3-87.0)

\section{E. nana}

No. of infected children

No. of children not cured after treatment

24

10

58.3

(37.1-79.6)

\section{C. mesnili}

No. of infected children

No. of children not cured after treatment

CR, \% (95\% Cl)

$\begin{array}{cc}17 & 10 \\ 4 & 1 \\ 76.5 & 90.0\end{array}$

(54.0-99.0)

(67.4-100.0)

$\begin{array}{cc}5 & 13 \\ 1 & 2 \\ 80.0 & 84.6\end{array}$

8

2

11

No. of infected children

No. of children not cured after treatment

CR, \% (95\% Cl)

$\mathrm{Cl}$, confidence interval; $\mathrm{CR}$, cure rate. 


\section{Discussion}

Since very little is known on the epidemiology of intestinal protozoa in sub-Saharan Africa, we analyzed formalinfixed stool samples obtained from 550 school-aged children who participated in a randomized controlled trial on Pemba Island to assess the efficacy and safety of nitazoxanide, albendazole and a combination of both drugs against T. trichiura and other soil-transmitted helminths. This trial found low CRs against soil-transmitted helminth species [19]. Importantly, the study provided an opportunity to shed new light on the extent of intestinal protozoa infections in a child cohort and to determine whether the different study treatments had an effect on intestinal protozoa species.

Our results confirm that intestinal protozoa are a public health issue on Pemba Island. Indeed, almost half of the children were infected with at least one of the three (potentially) pathogenic intestinal protozoa. When considering the results from the baseline and the 3-week post-treatment follow-up, assuming that a child who was diagnosed positive at follow-up was diagnosed as a false-negative case at baseline, the prevalence of intestinal protozoa infection was even higher (Figure 1). This issue is most likely explained by the lack of sensitivity when examining only a single stool sample with the ether-concentration technique, an important limitation of our study. Hence, in future studies, multiple stool samples should be examined and subjected to the ether-concentration method or more sensitive molecular approaches employed to improve diagnostic accuracy [26,27].

A recent study reported only moderate sensitivity for the ether-concentration technique compared to the FLOTAC technique when examining the same formalinfixed stool samples [28]. However, a study among five European reference laboratories showed that the agreement of diagnostic results was only moderate for pathogenic intestinal protozoa although the participating centres adhered to the same standard operating procedures for the ether-concentration technique [21]. In particular, E. histolytica is frequently misdiagnosed, even by experienced laboratory personnel [29].

We found that girls were generally at higher odds of an infection with any of the intestinal protozoa encountered than boys. Similar results were found by Mohammed Mahady and colleagues in Malaysia [30]. On the other hand, Traoré et al., in a study carried out in school-aged children in Côte d'Ivoire, reported a considerably higher prevalence of intestinal protozoa among boys than girls [31], corroborating findings by Cifuentes et al. from Mexico, where boys were at higher odds of a $G$. intestinalis infection that girls [32]. Other studies found no gender difference at all [33]. These findings indicate that intestinal protozoa infections may be related to gender-specific behaviour within a community. In addition, we observed statistically significant differences for some of the intestinal protozoa between the two schools. Hence, even though the schools are located only a few kilometers apart and the two settings were quite similar, at least in terms of socioeconomic status, availability of safe water supply and sanitation infrastructure, different infection profiles were observed. This highlights the possibility of 'micro-geographic' variability in endemicity of intestinal protozoa infection. One explanation could be that Al-Sadik school is located close to an orphanage, where transmission of intestinal protozoa might be enhanced. Other behavioural, infrastructure or environmental factors that may account for our observation should be investigated in future studies.

Intestinal protozoa often co-occur with intestinal nematodes and it is therefore important to determine whether anthelminthic and other antiparasitic drugs have an effect on concomitant intestinal protozoa infections. Albendazole, for example, was found in a recent meta-analysis to be as effective as metronidazole against G. intestinalis [34]. The observed CRs against all intestinal protozoa were moderate to high in the three treatment groups. However - and contrary to what we expected - there was also a moderate treatment efficacy in the placebo group, which is difficult to explain other than a diagnostic dilemma. Our findings therefore have to be interpreted with caution. The high 'cure rate' observed within the placebo group underscores that analysis of a single stool sample with the formalin-ether concentration technique is unreliable, and hence, the CRs for the three treatment schemes investigated here are likely overestimated. Still, our results show that none of the drugs administered as single dose resulted in cure of all infected children. Only moderate CRs were observed against G. intestinalis, E. histolytica/E. dispar and the potentially pathogenic $B$. hominis. A further limitation is the relatively small number of positive children for specific intestinal protozoa. Infection intensity did not significantly decrease after treatment with the exception of $E$. coli in the nitazoxanide group. Note that intestinal protozoa multiply within the host, which can also influence infection intensity results [35]. Further, the incubation time for different intestinal protozoa is between 7 and 28 days, meaning that it is possible that within the 3 -week period before and after treatment, re-infection had occured [36-40]. The clinical relevance of the moderate CRs suggests that single-dose albendazole or nitazoxanide or a combination of the two drugs do not have sufficient efficacy against pathogenic intestinal protozoa. However, multiple stool samples should be examined to strengthen the diagnostic accuracy for these infections. 


\section{Conclusion}

Our study revealed that intestinal protozoa infections are highly prevalent among school-aged children on Pemba Island, a setting where these intestinal protozoa were not considered of major importance thus far. The difficulty in accurately diagnosing intestinal protozoa infections, also experienced in our study, might be one of the chief reasons why these pathogens and the diseases they cause are often neglected, even in settings where mass treatment are underway against intestinal helminth infections [21]. The prevalence of intestinal protozoa is therefore unknown in many settings and consequently the disease burden cannot be fully quantified [41]. Accurate diagnostic tools that can be applied at the point-of-care are thus urgently required. Finally, drugs and effective treatment regimens for intestinal protozoa infections to be used in public health campaigns are still far out of reach.

\section{Competing interests}

None of the authors has any conflict of interest concerning the work reported in this paper.

\section{Authors' contributions}

BS, ShMA, SaMA, JU, MA and JK designed the study; BS, HM, ShMA, SaMA and JK implemented the study; BS managed the data; BS, HM, IB, JU and JK analyzed and interpreted the data; BS wrote the first draft of the paper; $H M$, ShMA, SaMA, IIB, JU, MA and JK revised the manuscript. All authors read, and approved the manuscript prior to submission and assisted with the final revision of the manuscript.

\section{Author details}

${ }^{1}$ Department of Medical Parasitology and Infection Biology, Swiss Tropical and Public Health Institute, P.O. Box, CH-4002 Basel, Switzerland. ${ }^{2}$ University of Basel, P.O. Box, CH-4003 Basel, Switzerland. ${ }^{3}$ Department of Medical and Diagnostic Services, Swiss Tropical and Public Health Institute, P.O. Box, CH-4002 Basel, Switzerland. ${ }^{4}$ Public Health Laboratory (Pemba)-Ivo de Carneri, P.O. Box, TZ-122 Wawi, Chake Chake, Tanzania. ${ }^{5}$ Divisions of Internal Medicine and Infectious Diseases, Toronto General Hospital, Toronto, Ontario, Canada. ${ }^{6}$ Department of Epidemiology and Public Health, Swiss Tropical and Public Health Institute, P.O. Box, CH-4002 Basel, Switzerland. ' Ivo de Carneri Foundation, P.O. Box, IT-10122 Milan, Italy.

Received: 13 December 2012 Accepted: 25 December 2012 Published: 4 January 2013

\section{References}

1. Feng $Y$, Xiao L: Zoonotic potential and molecular epidemiology of Giardia species and giardiasis. Clin Microbiol Rev 2011, 24:110-140.

2. Stanley SL: Amoebiasis. Lancet 2003, 361:1025-1034

3. Lozano R, Naghavi M, Foreman K, Lim S, Shibuya K, Aboyans V, Abraham J, Adair T, Aggarwal R, Ahn SY, et al: Global and regional mortality from 235 causes of death for 20 age groups in 1990 and 2010: a systematic analysis for the Global Burden of Disease Study 2010. Lancet 2012, 380:2095-2128.

4. Escobedo AA, Cimerman S: Giardiasis: a pharmacotherapy review. Expert Opin Pharmacother 2007, 8:1885-1902.

5. Jex AR, Smith HV, Nolan MJ, Campbell BE, Young ND, Cantacessi C, Gasser RB: Cryptic parasite revealed improved prospects for treatment and control of human cryptosporidiosis through advanced technologies. Adv Parasitol 2011, 77:141-173.

6. Davies AP, Chalmers RM: Cryptosporidiosis. BMJ 2009, 339:b4168.

7. Stensvold CR, Lewis HC, Hammerum AM, Porsbo LJ, Nielsen SS, Olsen KE, Arendrup MC, Nielsen HV, Mølbak K: Blastocystis: unravelling potential risk factors and clinical significance of a common but neglected parasite. Epidemiol Infect 2009, 137:1655-1663.
8. Leder K, Hellard ME, Sinclair MI, Fairley CK, Wolfe R: No correlation between clinical symptoms and Blastocystis hominis in immunocompetent individuals. J Gastroenterol Hepatol 2005, 20:1390-1394

9. Sheehan DJ, Raucher BG, McKitrick JC: Association of Blastocystis hominis with signs and symptoms of human disease. J Clin Microbiol 1986, 24:548-550.

10. Yoder JS, Harral C, Beach MJ: Giardiasis surveillance-United States, 20062008. MMWR Surveill Summ 2010, 59:15-25.

11. Hellard ME, Sinclair MI, Hogg GG, Fairley CK: Prevalence of enteric pathogens among community based asymptomatic individuals. J Gastroenterol Hepatol 2000, 15:290-293.

12. Taylor DN, Houston R, Shlim DR, Bhaibulaya M, Ungar BL, Echeverria P: Etiology of diarrhea among travelers and foreign residents in Nepal. JAMA 1988, 260:1245-1248.

13. Escobedo AA, Almirall P, Alfonso M, Cimerman S, Rey S, Terry SL: Treatment of intestinal protozoan infections in children. Arch Dis Child 2009, 94:478-482.

14. Rodriguez-Garcia R, Rodriguez-Guzman LM, Cruz del Castillo AH: Effectiveness and safety of mebendazole compared to nitazoxanide in the treatment of Giardia lamblia in children. Rev Gastroenterol Mex 1999, 64:122-126.

15. Yereli K, Balcioglu IC, Ertan P, Limoncu E, Onag A: Albendazole as an alternative therapeutic agent for childhood giardiasis in Turkey. Clin Microbiol Infect 2004, 10:527-529.

16. Ortiz JJ, Ayoub A, Gargala G, Chegne NL, Favennec L: Randomized clinical study of nitazoxanide compared to metronidazole in the treatment of symptomatic giardiasis in children from northern Peru. Aliment Pharmacol Ther 2001, 15:1409-1415.

17. Pampiglione S, Visconti S, Stefanini A: Human intestinal parasites in subSaharan Africa. III. Pemba Island (Zanzibar-Tanzania). Parassitologia 1987, 29:27-35 (in Italian).

18. Albonico M, De Carneri I, Di Matteo L, Ghiglietti R, Toscano P, Uledi MK, Savioli L: Intestinal parasitic infections of urban and rural children on Pemba Island: implications for control. Ann Trop Med Parasitol 1993, 87:579-583.

19. Speich B, Ame SM, Ali SM, Alles R, Hattendorf J, Utzinger J, Albonico M, Keiser J: Efficacy and safety of nitazoxanide, albendazole, and nitazoxanide-albendazole against Trichuris trichiura infection: a randomized controlled trial. PLoS Negl Trop Dis 2012, 6:e1685.

20. Marti H, Escher E: SAF-an alternative fixation solution for parasitological stool specimens. Schweiz Med Wochenschr 1990, 120:1473-1476 (in German).

21. Utzinger J, Botero-Kleiven S, Castelli F, Chiodini PL, Edwards H, Kohler N, Gulletta M, Lebbad M, Manser M, Matthys B, et al: Microscopic diagnosis of sodium acetate-acetic acid-formalin-fixed stool samples for helminths and intestinal protozoa: a comparison among European reference laboratories. Clin Microbiol Infect 2010, 16:267-273.

22. Diamond LS, Clark CG: A redescription of Entamoeba histolytica Schaudinn, 1903 (Emended Walker, 1911) separating it from Entamoeba dispar Brumpt, 1925. J Eukaryot Microbiol 1993, 40:340-344.

23. Pierce KK, Kirkpatrick BD: Update on human infections caused by intestinal protozoa. Curr Opin Gastroenterol 2009, 25:12-17.

24. Leitch GJ, He Q: Cryptosporidiosis-an overview. J Biomed Res 2012, 25:1-16.

25. Coulibaly JT, Fürst T, Silué KD, Knopp S, Hauri D, Ouattara M, Utzinger J, N'Goran EK: Intestinal parasitic infections in schoolchildren in different settings of Côte d'Ivoire: effect of diagnostic approach and implications for control. Parasit Vectors 2012, 5:135.

26. Marti H, Koella JC: Multiple stool examinations for ova and parasites and rate of false-negative results. J Clin Microbiol 1993, 31:3044-3045.

27. van Lieshout $L$, Verweij JJ: Newer diagnostic approaches to intestinal protozoa. Curr Opin Infect Dis 2010, 23:488-493.

28. Becker SL, Lohourignon LK, Speich B, Rinaldi L, Knopp S, N'Goran EK, Cringoli G, Utzinger J: Comparison of the Flotac-400 dual technique and the formalin-ether concentration technique for diagnosis of human intestinal protozoon infection. J Clin Microbiol 2011, 49:2183-2190.

29. Rayan HZE: Microscopic overdiagnosis of intestinal amoebiasis. $J$ Egypt Soc Parasitol 2005, 35:941-951.

30. Mohammed Mahdy AK, Surin J, Wan KL, Mohd-Adnan A, Hesham AlMekhlafi MS, Lim YAL: Giardia intestinalis genotypes: risk factors and correlation with clinical symptoms. Acta Trop 2009, 112:67-70. 
31. Traoré SG, Odermatt P, Bonfoh B, Utzinger J, Aka ND, Adoubryn KD, Assoumou A, Dreyfuss G, Koussémon M: No Paragonimus in high-risk groups in Côte d'Ivoire, but considerable prevalence of helminths and intestinal protozoon infections. Parasit Vectors 2011, 4:96.

32. Cifuentes $E$, Suarez $L$, Espinosa $M$, Juarez-Figueroa L, Martinez-Palomo A: Risk of Giardia intestinalis infection in children from an artificially recharged groundwater area in Mexico City. Am J Trop Med Hyg 2004, 71:65-70.

33. Prado MS, Strina A, Barreto ML, Oliveira-Assis AM, Paz LM, Cairncross S: Risk factors for infection with Giardia duodenalis in pre-school children in the city of Salvador, Brazil. Epidemiol Infect 2003, 131:899-906.

34. Solaymani-Mohammadi S, Genkinger JM, Loffredo CA, Singer SM: A metaanalysis of the effectiveness of albendazole compared with metronidazole as treatments for infections with Giardia duodenalis. PLOS Negl Trop Dis 2010, 4:e682.

35. Wright SG: Protozoan infections of the gastrointestinal tract. Infect Dis Clin North Am 2012, 26:323-339.

36. Rendtorff RC: The experimental transmission of human intestinal protozoan parasites. II. Giardia lamblia cysts given in capsules. Am J Hyg 1954, 59:209-220.

37. Rendtorff RC, Holt CJ: The experimental transmission of human intestinal protozoan parasites. IV. Attempts to transmit Endamoeba coli and Giardia lamblia cysts by water. Am J Hyg 1954, 60:327-338.

38. Jokipii AM, Hemilä M, Jokipii L: Prospective study of acquisition of Cryptosporidium, Giardia lamblia, and gastrointestinal illness. Lancet 1985, 326:487-489.

39. Katz M, Despammier DD, Gwadz RW: Parasitic Diseases. New York, N.Y: Springer; 1989

40. Garcia LS, Bruckner DA: Diagnostic Medical Parasitology. 3rd edition. Washington, D.C.: ASM Press; 1997.

41. Okhuysen PC: Traveler's diarrhea due to intestinal protozoa. Clin Infect Dis 2001, 33:110-114.

doi:10.1186/1756-3305-6-3

Cite this article as: Speich et al:: Prevalence of intestinal protozoa infection among school-aged children on Pemba Island, Tanzania, and effect of single-dose albendazole, nitazoxanide and albendazolenitazoxanide. Parasites \& Vectors 2013 6:3.

\section{Submit your next manuscript to BioMed Central and take full advantage of:}

- Convenient online submission

- Thorough peer review

- No space constraints or color figure charges

- Immediate publication on acceptance

- Inclusion in PubMed, CAS, Scopus and Google Scholar

- Research which is freely available for redistribution 